\title{
SINGLE-SITE MAGNETOTELLURIC RESPONSE FUNCTIONS USING B-ROBUST W-ESTIMATORS, WITH AN APPLICATION TO EARTHQUAKE PREDICTION RESEARCH
}

\author{
A. TZANIS ${ }^{1}$
}

\begin{abstract}
The accumulation of stress and strain is known to induce changes in the electrical properties of rocks, which can be monitored for signs of earthquake preparation processes. To this effect, the Magnetotelluric sounding method presents some unique advantages. However, single-site MT data are notoriously susceptible to natural or anthropogenic time-varying coherent noise, which may severely bias the response function estimators and degrade their repeatability, unless treated with dedicated processing techniques. Such a technique is presented herein, involving the W-estimator with random error weighting, followed by an iterative robustification scheme based on an influence function approach. The algorithm is demonstrated on a set of severely distorted data exhibiting a marginal distribution of outliers, and is shown to effectively reduce the bias errors and the variance. It is also applied to the long-term monitoring of crustal resistivity with MT response functions at a noisy site located near Aerino village, SE Thessaly, Greece, achieving a sustainable repeatability threshold of 10-20\% and faring very well with respect to the data quoted from the international literature.
\end{abstract}

KEY WORDS: Earthquake Prediction, Magnetotellurics, Impedance Tensor, Robust Estimation, W-estimator.

\section{INTRODUCTION}

Earthquake preparation processes are thought to produce long and short term changes in the electrical properties of rocks, for instance through the mechanisms described by volume dilatancy models (Scholz, 1990; Myachkin et al., 1975). Electromagnetic (EM) fields may sense such changes, thereby offering a means of searching for earthquake premonitory phenomena. Active measurements with dc fields were frequently reported to have detected precursory resistivity changes and a large body of literature may be found in the reviews of Park et al., (1993) and Johnston, (1997). Passive measurements of ELF-ULF Magnetotelluric (MT) fields are not as successful. A respectable volume of earlier work has been reviewed by Beamish (1982) and Kharin (1982), but not much progress has been made since, (for instance, see Johnston, 1997), with Ernst et al., (1993), Rozluski and Yukutake (1993) and Svetov et al., (1997) deserving attention. With the exception of Svetov et al. (1997), not one of the references cited in the international literature uses MT response functions, (namely the MT impedance tensor), which have yet to find their place in the arsenal of earthquake prediction research.

The hold up is due to the susceptibility of ELF-ULF MT data to coherent natural and anthropogenic noise, which may severely degrade the quality and long term stability of the response functions. Natural noise derives from phenomena such as time-dependent streaming and $\zeta$ potentials, nearby lightning etc. Anthropogenic noise derives from the operation of electric and electronic devices. A balanced power distribution grid will generate a fundamental at $50 / 60 \mathrm{~Hz}$ and discrete higher order harmonics, easy to rid of. An unstable grid, however, produces modulation and wide band interference, much harder to deal with. Transient noise is generated by the switching of machines and may propagate over long distances. It appears in the form of step functions, boxcar functions, spiky or dispersed $\delta$-functions, damped quasi-sinusoids etc., debilitating a broad-band spectrum. These types of noise may cause serious, even insurmountable problems to single-site data and cannot be treated with standard methods, as will be

1. Department of Geophysics and Geothermy, University of Athens, Zografou 157 84, Greece; atzanis@cc.uoa.gr. 
seen below. Until very recently, even by using remote referencing systems to obtain consistent and repeatable response functions, it has proved difficult to reduce errors below several percent for good data and very much higher for poor data. Inasmuch as precursory resistivity changes are expected (and are observed) to be only a few percent, it is easy to see why progress has been slow.

Nevertheless, the MT fields are unique in their capacity to probe a broad depth range with passive point measurements on the surface. Moreover, MT response functions may be used to discriminate possible transient telluric precursors, by deconvolving the induced from the total observed electric field, as in Arvidsson and Kulhanek, (1993), or Tzanis, (1994). Last but not least, MT is a unique crustal sounding method, with a wide range of academic and commercial applications. Therefore, it is compelling to research for methods of improving the reliability and stability of the response functions.

Noise reduction schemes for single-site data have been devised by several researchers. The earlier efforts included variants of the W-estimator (e.g. Jones et al., 1983; Beamish, 1986). Later work concentrated on robust-resistant methods, (e.g. Egbert and Booker, 1986; Chave et al., 1987; Larsen, 1989; Sutarno and Vozoff, 1991; Egbert and Livelybrooks, 1996), using some form of the regression-M estimator. of all these approaches, the W-estimator is the simplest and most versatile and, as will be shown, very powerful when combined with the right weighting schemes. Moreover, it can be robustified to cope with data containing tailed marginal error distributions and outliers. Such an algorithm has been developed by Tzanis (1988) and herein is adapted to the frequency domain estimation of MT responses.

The algorithm is applied to the analysis of MT data from an earthquake prediction experiment in SE Thessaly, Greece, involving long term observations of ULF natural electromagnetic fields. This region exhibits moderate seismicity and considerable earthquake hazard (e.g. Kouskouna, 1991). During the $20^{\text {th }}$ century only, the area experienced eight main sequences, with twelve shallow earthquakes having magnitudes $\mathrm{M}_{\mathrm{s}}$ $>6(1905,1911,1930,1941,1954,1955,1957,1980)$. Of these, all the post-1954 earthquakes occurred within the rectangle $22.5^{\circ} \mathrm{E}-23.3^{\circ} \mathrm{E}$ and $39^{\circ} \mathrm{N}-39.5^{\circ} \mathrm{N}$ in the periphery of Volos, Velestino and Almyros cities. This area is characterised by rather infrequent, albeit large earthquakes separated by decade-long periods of quiescence, during which it might be possible to observe stress-and-strain induced changes in the electric properties of rocks.

\section{FREQUENCY DOMAIN MT RESPONSE FUNCTION ESTIMATION}

The conventional approach to single-site frequency domain Magnetotelluric impedance tensor estimation is based on the assumption of stochastic (Gaussian) time processes. The horizontal electric and magnetic field components are measured simultaneously in the time domain and in two, mutually orthogonal Cartesian frames. Following transformation into the frequency domain, the most common method for estimating the impedance tensor elements $z_{i j}, i=x, y$, is the least squares solution of the two-input one-output linear system

$$
E_{i}=Z_{i x} H_{x}+Z_{i y} H_{y}+\varepsilon_{i}, \quad i=x, y,
$$

by minimising the noise $\varepsilon_{i}$ (Sims et al., 1971). This yields a system of two equations in two unknowns,

$$
E_{i} F_{j}^{*}=Z_{i x}\left(H_{x} F_{j}^{*}\right)+Z_{i y}\left(H_{y} F_{j}^{*}\right), \quad i=x, y, \quad j=x, y
$$

When $\mathrm{F} \equiv \mathrm{H}$, the solution is biased downwards by noise in the auto-spectra of the magnetic channels only. When F $\equiv E$ the solution is biased upwards by noise in the autospectra of the electric channels only. The quality of the solution can be monitored by means of the predicted coherence function

$$
\gamma_{i .23}^{2}=\mathrm{Mi} / \mathrm{Ei}, \quad \mathrm{Mi}=\bullet \mathrm{ixHx}+\cdot \mathrm{iyHy}_{\mathrm{H}} \quad \mathrm{i}=\mathrm{x}, \mathrm{y},
$$

where $M_{i}$ represents the output electric field component predicted from the estimated impedance elements ${ }_{i j}$. Clearly, $0 \leq \gamma^{2} \leq 1$, so that low values of the predicted coherence 
indicate a rejectable solution and high values an acceptable solution. Given $\mathrm{N}$ spectral realizations of $\mathrm{E}_{\mathrm{x}}, \mathrm{E}_{y}, \mathrm{H}_{\mathrm{x}}$ and $\mathrm{H}_{y}$ at a frequency $\omega$, it is necessary to provide at least two limiting cases of noise content, namely a population $\left\{\mathrm{O}_{i j}\right\}$ of stable, downwards biased estimates and a population $\left\{\mathrm{U}_{i j}\right\}$ of stable, upwards biased estimates. If the data provides an adequate distribution of high predicted coherences, the bias errors reduce to the magnitude of random errors. Each of $\left\{\mathrm{O}_{i j}\right\}$ and $\left\{\mathrm{U}_{i j}\right\}$ are used to calculate the expectation values $\left\langle\mathrm{O}_{i j}\right\rangle$ and $\left\langle\mathrm{U}_{i j}\right\rangle$ and variances $\Delta \mathrm{O}_{i j}$ and $\Delta \mathrm{U}_{i j}$. The 'true' values of the impedance tensor $<\bullet_{i j}>$ elements can, then, be estimated from the lower and upper bounds. In the case of Gaussian (stationary) noise, straightforward averages of $\left\{\mathrm{O}_{i j}\right\}$ and $\left\{\mathrm{U}_{i j}\right\}$ would suffice to provide the final unbiased elements. Unfortunately, the noise is usually non-stationary and quite often coherent across channels (multiple coherent noise), evading the predicted coherence test, and introducing tailed marginal error distributions and outliers, into $\left\{\mathrm{O}_{i j}\right\}$ and $\left\{\mathrm{U}_{i j}\right\}$, thereby providing biased and oscillatory estimates $\left\langle\boldsymbol{\bullet}_{i j}\right\rangle$. In the following, I will first attempt clarify the concepts and then explain the robust W-estimation procedures developed to deal with such noise.

At frequency $\omega$, the $\mathrm{N}$ realizations of equation (1) can be cast into an overdetermined system of $\mathrm{N}$ equations in 2 unknowns, denoted by $\mathbf{E}_{\mathrm{N}}=\mathbf{H}_{\mathrm{N}} \mathbf{z}$. Alternatively, one can arrange in a $2 \mathrm{~N} \times 2$ matrix, $\mathrm{N}$ sub-systems of the form (2), generating an overdetermined system of $2 \mathrm{~N}$ equations in 2 unknowns denoted by $\mathbf{E}_{2 \mathrm{~N}}=\mathbf{H}_{2 \mathrm{~N}} \mathbf{z}$. Then, one seeks to obtain a maximum likelihood estimate of $\mathbf{z}$ by minimising an expression of the form

$$
\sum_{n} \rho\left(r_{n}\right)=\sum_{n}\left(E_{n}-H_{n} \mathbf{z}\right) / \sigma, \quad n=1,2, \ldots, m N
$$

with $m=1$ or 2 , where $\rho(r)$ is a suitable loss function, $r_{n}$ is the residual of the $n^{\text {th }}$ observation and $\sigma$ is a normalising error scale factor. For instance, a loss function $\rho(r)=r^{2} / 2$ leads to the minimization of the $L_{2}$ norm

$$
\Sigma_{n} r_{n}^{2}=\Sigma_{n}\left(E_{n}-H_{n} \mathbf{z}\right)^{2}
$$

and corresponds to the standard least squares (LS) solution. In the presence of biasing non-Gaussian noise, we seek to define a maximum likelihood estimator (Mestimator) of the location of $\mathbf{z}$, which must show minimal bias (B-robust) and minimal change of variance (V-robust). This approach to M-estimation usually requires the solution of a non-linear system of equations, but this can be avoided with iterative procedures involving the so-called influence function $\psi(r)$ and weight factors of the form $w(\psi(r))$. The influence function is generically related to the loss function, usually as $\psi(r)=\rho^{\prime}(r)=d \rho(r) / d r$. In general, one obtains an initial estimate $\bullet(0)$. Then, looping over $K$ iterations to convergence, one calculates the predicted output $M_{n}(k)=H_{n}(k) \cdot(k)$, calculates $r_{n}(k)=E_{n}(k)-M_{n}(k), \psi\left(r_{n}(k)\right)$ and $w\left(\psi\left(r_{n}\right)\right)$, modifies the output as $\mathrm{E}_{\mathrm{n}}(\mathrm{k}+1)=\mathrm{M}_{\mathrm{n}}(\mathrm{k})+\mathrm{w}(\psi) \mathrm{r}_{\mathrm{n}}(\mathrm{k})$ and obtains new solutions $\cdot(\mathrm{k}+1)$ and a new error scale $\sigma(k+1)$. The successive approximations $\bullet(k)$ are usually obtained with LS algorithms. This is the regression-M estimation, for which one may seek details in Huber (1981) and Hampel et al., (1986).

Now, let $\mathrm{Z} \equiv \mathrm{Z}_{i j}$ define any element, and $\mathbf{z}=\left[\mathrm{Z}_{i x} \mathrm{Z}_{i y}\right]$ any row of the tensor. The Westimator is defined as a weighted average $\langle\mathrm{Z}\rangle$ of the observations

$$
\mathrm{Z}\left\{\mathrm{N}: \mathrm{Z}_{1}, \mathrm{Z}_{2}, \ldots, \mathrm{Z}_{\mathrm{N}}\right\}=\sum_{n} \mathrm{~W}_{n} \cdot \mathrm{Z}_{n} \cdot\left[\sum_{n} \mathrm{~W}_{n}\right]^{-1}
$$

with the weights depending on the observations according to $W_{n}=W\left(Z_{n}-Z\right)$ and being functions of the location of $z_{n}$ within the sample space. Therefore, the W-estimator satisfies the equation

$$
<Z>=\sum_{n} W\left(z_{n}-<Z>\right) \cdot Z_{n} \cdot\left[\sum_{n} W\left(z_{n}-<Z>\right)\right]^{-1}
$$

which can be modified to yield

$$
0=\sum_{n} W\left(Z_{n}-\langle Z>) \cdot\left(Z_{n}-<Z>\right) \cdot\left[\sum_{n} W\left(Z_{n}-<Z>\right)\right]^{-1}\right.
$$

implying that

$$
0=\sum_{n} \rho\left(z_{n}-<z>\right) \quad \text { with } \rho^{\prime}\left(z_{n}-<z>\right)=\left(z_{n}-<Z>\right) \cdot W\left(z_{n}-<Z>\right) \text {, }
$$


where $\rho$ is just another expression for the general form of a loss function with the error scale omitted, since $\langle\mathrm{Z}>=\mathrm{E}\{\mathrm{Z}(\mathrm{N})\}$. This means that the iterated $\mathrm{W}$-estimator is a variant of the M-type estimators of location. In fact it can be understood as a one-step regression-M estimator and as such, it possesses the same influence function and asymptotic variance (Hampel et al., 1986).

In order to construct a B-robust W-estimator for MT response functions, consider the loss and influence functions

$\rho(r)=\left\{\begin{array}{rl}\frac{1}{2} r^{2}, & |r|<r_{0} \\ r_{0}|r|-\frac{1}{2} r^{2}, & |r|>r_{0}\end{array}, \quad \psi(r)=\rho^{\prime}(r)=\left\{\begin{array}{r|l}r_{1} & \mid r k r_{0} \\ r_{0}, & \mid r p r_{0}\end{array}\right.\right.$.

with weights $w(r)=\psi(r) /|r|$ and $r_{0}=\alpha s$, where $s$ is the standard deviation of the uncontaminated error distribution and $\alpha$ is a real constant. This is a hybrid corresponding to $L_{2}$ minimisation for the small residuals and $L_{1}$ minimisation for the larger ones. The influence function is non-decreasing, which is a requirement for convergence to unique estimates. The weight function $w(r)$ is continuous and downweights outliers without break points other than $r_{0}$. General convergence is guaranteed, provided that $\rho(0)=0$, $\rho^{\prime}(r)>0$ and $0 \leq \rho(r) \leq 1$, (Huber, 1981).

Next, consider that one can always solve $N$ systems of the form (2), to obtain populations $\bullet\left(\mathrm{N}: \bullet_{1}, \bullet_{2}, \ldots, \bullet_{\mathrm{N}}\right)$, each estimate in the population exactly satisfying one of the $\mathrm{N}$ finite data realisations. This can be used to compute the successive approximations $\langle\cdot(k)>$ to $E\{Z(N)\}$, using the one-step W-estimator. Thus, it is not necessary to set up and solve any large over-determined system and the robust W-estimation reduces to an iterative re-weighted LS scheme as follows: 1) Obtain initial estimates of $\langle\bullet(0)\rangle$ and of the error scale $\sigma(0)$. 2) Using equation (2), compute the predicted output $M_{n}(k)=\mathbf{H}_{n}\langle\bullet(k)\rangle$ and the corresponding residual $r_{n}(k)=E_{n}(k)-M_{n}(k)$. 3) Modify the output as $M_{n}(k+1)=M_{n}(k)+w(r) \cdot r_{n}(k)$. 4) Use the modified observations and equation (2) to compute $N$ estimates $\bullet_{n}(k+1)$. 5) Form the W-estimators

$$
<\cdot(k+1)\rangle=\sum_{n} \cdot{ }_{n}(k+1) \cdot W_{n}(k+1) \cdot\left[\sum_{n} W_{n}(k+1)\right]^{-1}
$$

for each element of - and compute the new RMS error scale $\sigma(k+1)$, then iterate from 2 until convergence. The error scale can be computed as

$$
\sigma(k)=\left[(\beta(2 N-4))^{-1} \cdot \Sigma_{n} r_{n}(k)\right]^{1 / 2}
$$

i.e. is a scaled RMS error with $\beta<1$ so that the error scale will not be underestimated during the successive iterations. Egbert and Booker (1986) give a method to obtain the appropriate $\beta$ for any choice of $r_{0}$. The form of the weight factors $W_{n}$ is also (and apparently) important.
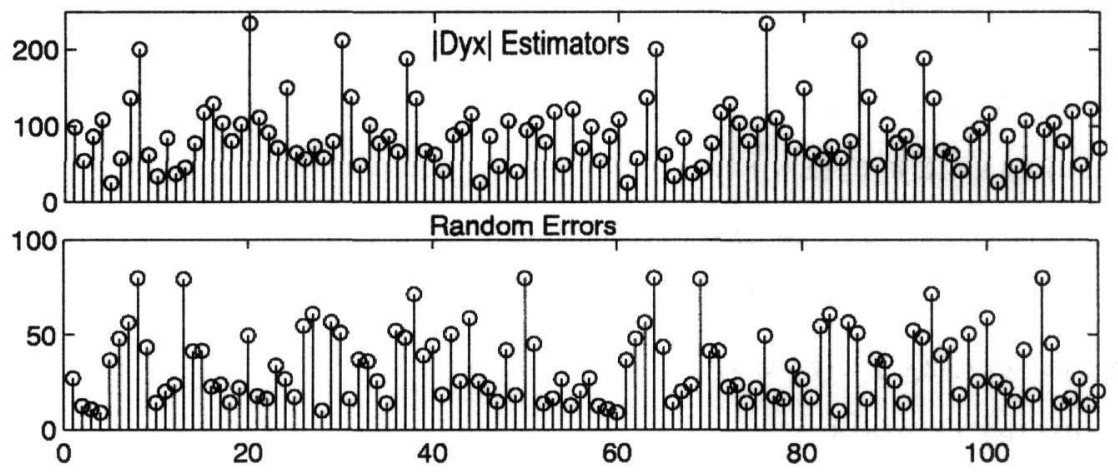

Figure 1. An ensemble of $\left\{D_{y x}\right\}$ impedance tensor elements (top) and their random errors (bottom). 

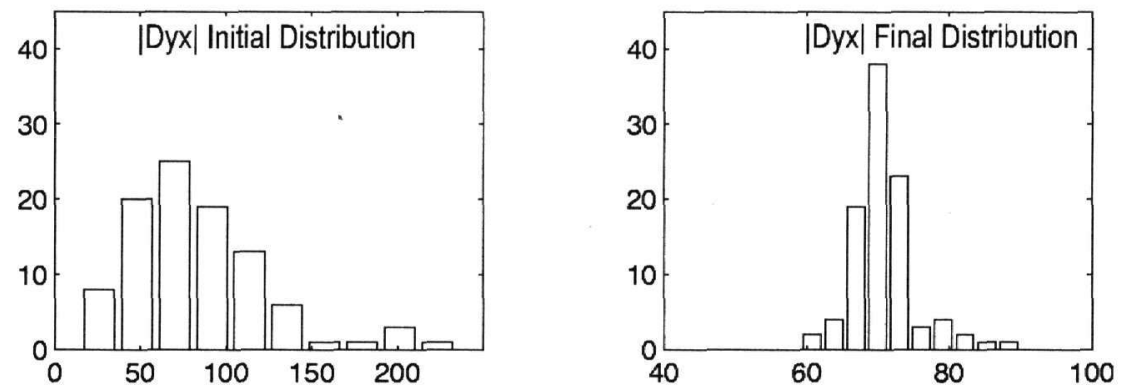

Figure 2. Left: The distribution of the original $\left|D_{y x}\right|$ population illustrated in Figure 1. Right: The final distribution of the processed ensemble, after application of the robust W-estimator (see Figure 3).

One obvious choice is to use the influence weight $w(r)$, which has the functional form

$$
W\left(r_{n}(k)\right)=f\left(M_{n}(k)-H_{n} \cdot(k)\right)=f\left(H_{n} \cdot\left[\cdot{ }_{n}(k)-\bullet(k)\right]\right)
$$

and, therefore, is a genuine function of the location of $\bullet_{n}(k)$. This weight function is very stable and will always ensure convergence.

\section{EXAMPLES}

It is quite apparent that the effectiveness of the $\mathrm{W}$-estimator depends on the choice of the weight function. The predicted coherence has often been used, (e.g. Jones et al., 1983; Egbert and Livelybrooks, 1996), but I have found, that Pedersen's (1982) random error

$$
\varepsilon^{2}=\begin{gathered}
1 \\
v-4 F_{1, v-4: a}\left(1-\gamma_{i, 23}^{2}\right)\left(E_{i} E_{i}^{*}\right) \\
\left(1-\gamma_{23}^{2}\right)\left(H_{j} H_{j}^{*}\right)
\end{gathered}, \quad i=x, y, \quad j=x, y,
$$

is very effective and quite robust (e.g. Tzanis 1988). In equation (4), $v$ is the number of degrees of freedom of the measured spectra, $F$ is the 100 a percentage point of an $F$ distribution with $v-4$ and 1 degrees of freedom and $\gamma_{23}$ is the coherency of $H_{x}$ and $H_{y}$. There are very sparse references to the properties of the random errors and their usefulness. The original study of Pedersen (1982), did not provide practical examples and to the best of my knowledge, only Beamish (1986) and Tzanis and Beamish (1989), report their implementation as weights, without giving details. Their efficiency however, is straightforward to demonstrate.

Figure 1 illustrates a population $\left\{\left|O_{y x}\right|\right\}$ above a predicted coherence threshold of 0.8 , contaminated by multiple-coherent non-Gaussian noise producing a heavily tailed marginal error distribution (Figure 2, left). It is clear that some estimators may display large deviations from the expectation values, but it is also quite apparent that all the outliers are invariably associated with large random errors, usually due to the high power of the noisy electric field. The population mean is $\left\langle 0_{y x}\right\rangle=79.58+i 13.98 \mathrm{mV} /$ $\mathrm{km} \cdot \mathrm{nT}$ and a W-estimator with predicted coherence weighting does not fare better for obvious reasons (only coherent estimates enter the population). The W-estimator with random error weighting yields $<0_{y x}>=68.28+i 16.05 \mathrm{mV} / \mathrm{km} \cdot \mathrm{nT}$ and significantly reduces the bias error. Thus, the random error emerges as a potentially useful diagnostic aid and data processor.

Figure 3 shows the robust iterated W-estimator operating on a subset of the same data series, (97 estimators of with predicted coherence above 0.85$)$, setting $<0_{y x}(0)>=68.28+i 12.05$ and using for weights $w_{n}(k)=w\left(r_{n}\right)=\psi\left(r_{n}\right)\left|r_{n}\right|^{-1}, r_{0}=1.5$ and $\beta=0.45$. The algorithm stops automatically after the second decimal point is fixed, yielding $<\mathrm{O}_{y x}>=68.28+i 19.80$. As can be seen, it eliminates the outliers and pushes the data towards the expectation value, allowing the corrected population to yield stable (Brobust) means with minimal variance (V-robust). Moreover, it converts the original broad, skewed and tailed distribution, to almost Gaussian (Figure 2, right). It is apparent that the estimate obtained by the iterated robust W-estimator did not improve the starting value by a significant factor, a fact that confirms the power of random error weighting. 

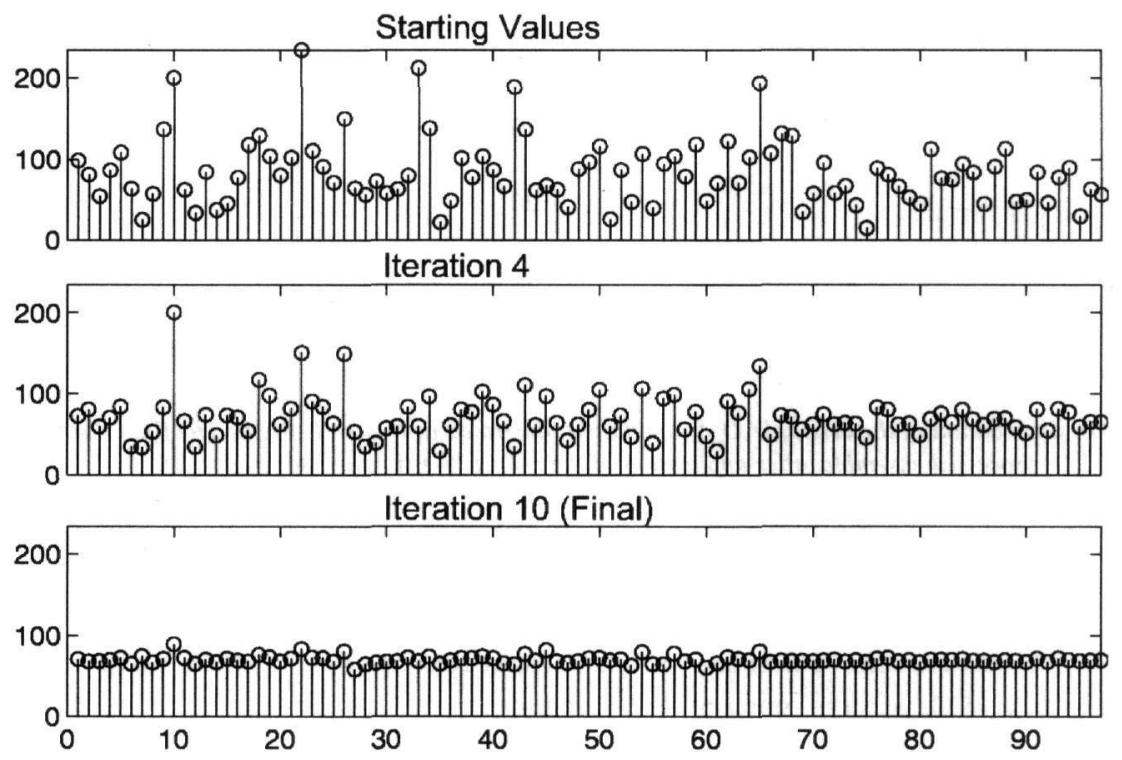

Figure 3. Application of the robust iterated $W$-estimator to the $Z_{y x}$ ensemble of Figure 1.

This algorithm will effectively downweight the influence of non-Gaussian noise, provided that the population of noise-free data dominates the population of noisy data. Its performance is a function of noise and data statistics, progressively deteriorating as the probability of receiving noise waveforms increases, until breakdown when the noise is as likely as the data and can cloak the distribution of the noise-free population beyond recognition and recovery. In this event, the treatment must be case-specific. The robust methods cannot cope with continuous harmonic multiple coherent noise, for obvious reasons. In conclusion, the robust W-estimator can provide effective means of overcoming non-Gaussian noise and can be useful for the long-term monitoring of time-dependence in the MT response functions.

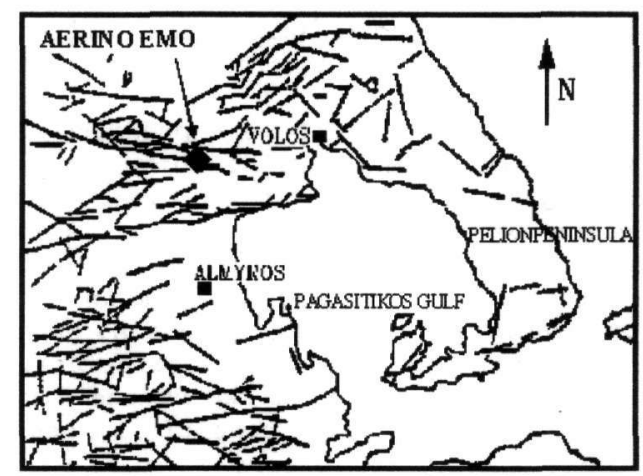

Figure 4. Location of the Aerino EMO and the tectonic lineaments in SE Thessaly, after Cratchley (1983).

\section{APPLICATION TO EARTHQUAKE PREDICTION RESEARCH}

The magnetotelluric data used in this study was recorded at a semi-permanent Electromagnetic observatory (EMO) located at an altitude of $210 \mathrm{~m}$ and at co-ordinates $39^{\circ} 21.5^{\prime} \mathrm{N}$ and $22^{\circ} 45.2^{\prime} \mathrm{E}$, near the village Aerino of Velestino county, SE Thessaly (Figure 4). The station was located on the hanging wall of the Chalkodonio mountain fault, above the seismogenic volume of the three Ms 6-6.8 events of 1957. Observations were carried out with non-polarising solid solution $\mathrm{Pb}-\mathrm{PbCl} 2$ electrodes and a $3-$ component fluxgate magnetometer. The data was recorded with a dedicated automatic 7channel logging unit, equipped with 20-bit $\delta$ - $\sigma$ digitisers and capacious disks, capable 
for extended autonomous operation. The observations were interrupted between August and November 1994, due to a brush fire that crippled the electric field sensor system, but fortunately leaving unscathed the other equipment, which was better protected. The EMO operated normally until July 1995 when the logger malfunctioned and was decommissioned. Response functions cannot be estimated at periods shorter than approx. 50s, due to the low sensitivity of the magnetometer (resolution > 0.01nT), and the continuously increasing noise levels from the expansion of Aerino village and nearby small agricultural industries. The results for the longer periods are estimated over fortnight to month long intervals and are presented in Figures $5 \mathrm{a}$ and $5 \mathrm{~b}$ for two distinct periods and in Figure 6 for the entire spectrum of the off-diagonal tensor elements $z_{x y}$ and $z_{y x}$. In general, long term magnetotelluric crustal monitoring with response functions repeatable to within 10-20\% has been possible. As can be observed in Figure 5, the shorter periods (higher frequencies) are estimated with better repeatability, within approximately 10\%, than the longer periods, (lower frequencies), which are estimated with a repeatability of $15 \%$ or higher. These differences are attributed to the different data and noise statistics at different parts of the spectrum but overall, the technique performs very well compared to the results quoted from the international literature for single-site long period data, (e.g. Park et al., 1993; Johnston, 1997). At any rate, the available data indicate that no systematic observable changes in the deep (lower crustal) geoelectric structure have taken place during October 1993 - July 1995. Nevertheless, long period (>50s) data may well miss the better part of the schizosphere, where major earthquakes nucleate (10-15 km). Shorter period observations with sensitive induction coils are likely to provide more meaningful information on processes occurring at depths comparable to the strongly deforming volumes of intermediate - large earthquakes.

AERINO BASE. TEST PERIOD: $90.0000 \mathrm{sec}$

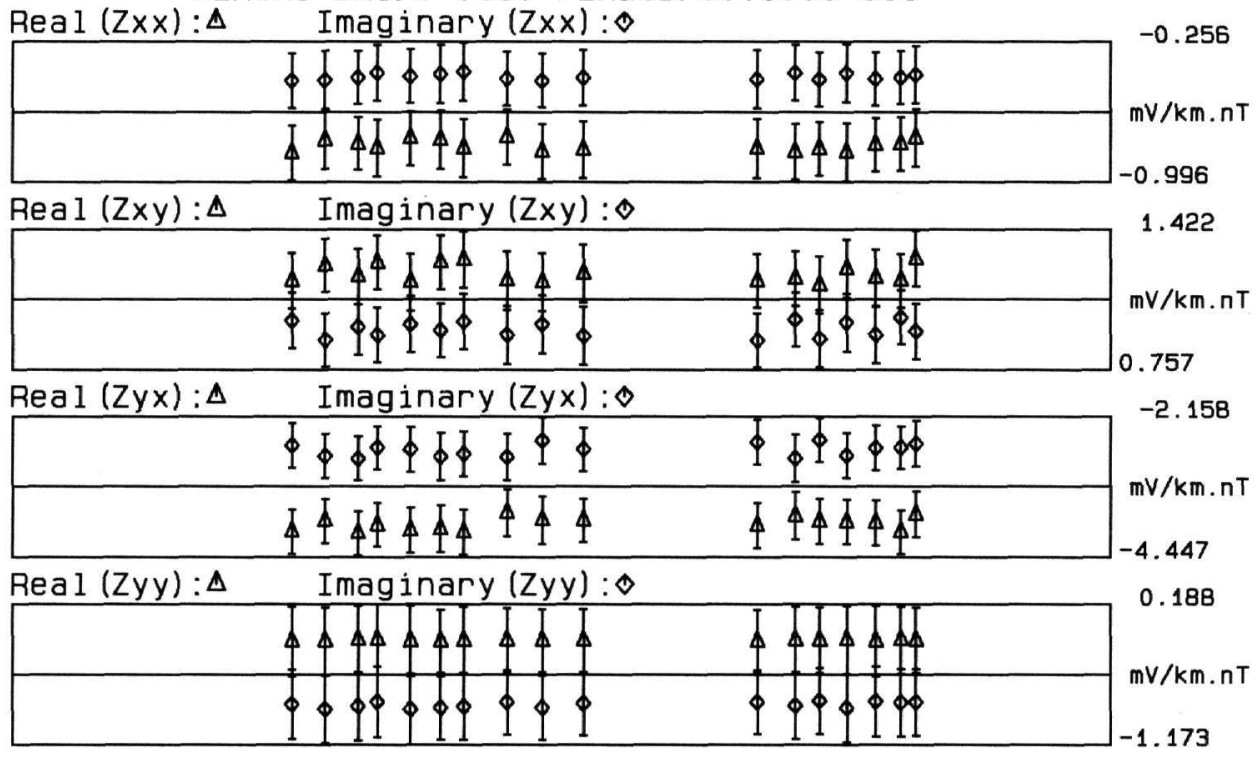

123456789101112123456789101112123456789101112 1993

1994 1995

Elapsed Time in Months

Figure 5a. Time dependence of the MT impedance tensor at $T=90$ s. 
AERINO BASE. TEST PERIOD: $5120.0000 \mathrm{sec}$

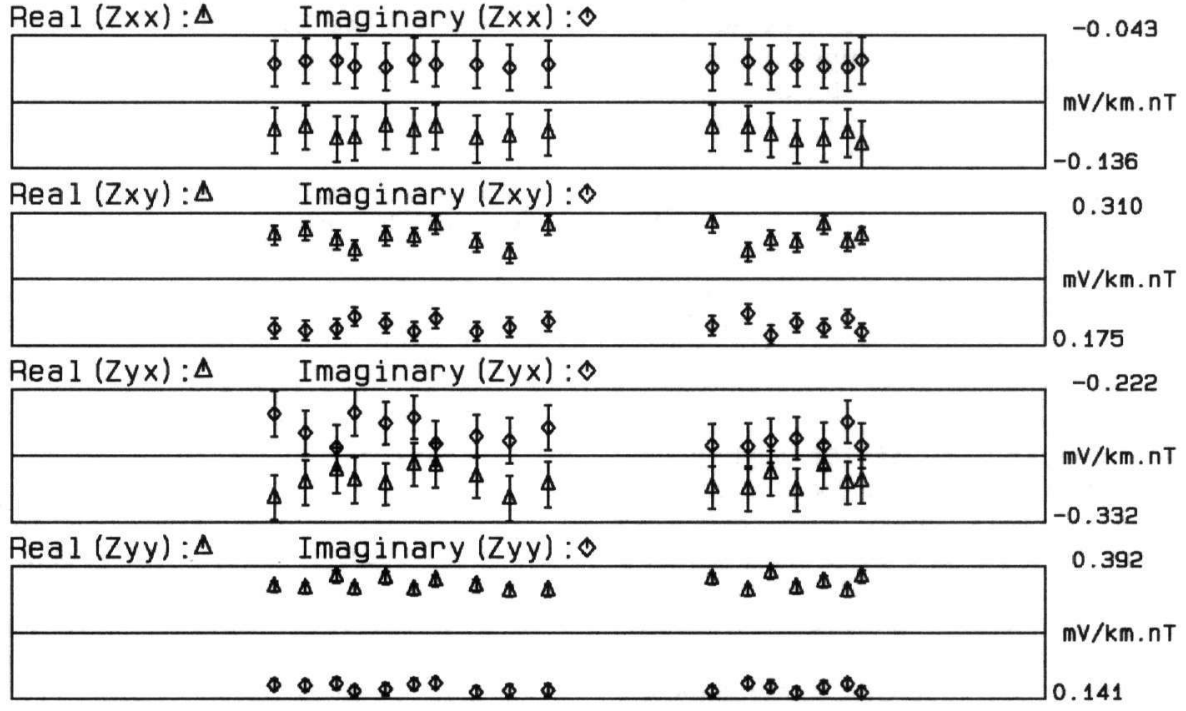

123456789101112123456789101112123456789101112
1993
1994
Elapsed Time in Months

Figure $5 b$. Time dependence of $M T$ impedance tensor at $T=5120 \mathrm{~s}$.
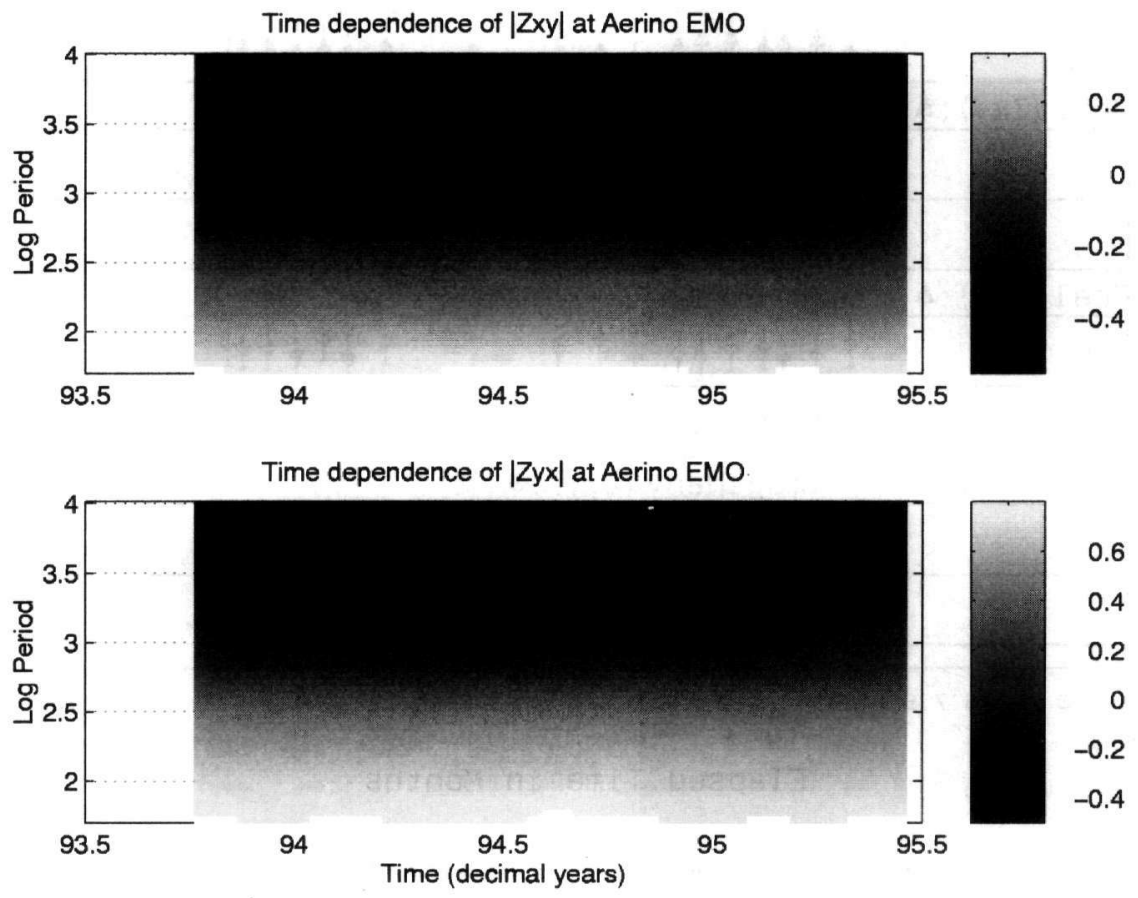

Figure 6. The time dependence of broad-band off-diagonal impedance thesor elements at the Aerino EMO. 


\section{ACKNOWLEDGEMENTS}

The Electormagnetic Observatory at Aerino was financially supported by the European Commission programmes EPOCH-CT91-0045 and EV5V-CT94-0449. Sincere thanks to Prof. Dr G-A Tselentis and to Dr P. Karmis for their constructive reviews.

\section{REFERENCES}

ARVIDSSON, R. AND KULHANEK, O., 1993. Enhancement of seismic electric signals using magnetotellurics, Tectonophysics, 224, 131-139.

BEAMISH, D., 1982. The time dependence of electromagnetic response functions. Geophysical Surveys, 4, $405-434$.

BEAMISH, D., 1986. Deep geoelectric structure beneath the Northumberland Basin, Geophys. J. R. astr. Soc., 84, 619-640.

CHAVE, A.D., THOMSON, D.J. AND ANDER, M.E., 1987. On the robust estimation of power spectra, coherences and transfer functions, J. Geophys. Res., 92, 633-648.

CRATCHLEY, C.R., 1983. Volos Project, PA 85F. Progress report, IGS component for the period 1st April-30 September 1983 , British Geological Survey.

EGBERT, G.D. AND BOOKER, J.R., 1986. Robust Estimation of Geomagnetic Transfer Function Data, Geophys. J. R. astr. Soc., 87, 173-194.

EGBERT, G.D. AND LIVELYBROOKS, D.W., 1996. Single station magnetotelluric impedance estimation: Coherence weighting and the regression M-estimate, Geophysics, 61, 964-970.

ERNST, T., JANKOWSKI, J., ROZLUSKI, C. AND TESSEYRE, R., 1993. Analysis of the electromagnetic field recorded in the Friuli seismic zone, NE Italy, Tectonophysics, 224, 141-148.

HAMPEL, F.R., RONCHETTI, E.M., ROUSSEEUW, P.J. AND STAHEL, W.A., 1986. Robust Statistics: An Approach Based on Influence Functions, Wiley.

HUBER, P.J., 1981. Robust Statistics, Wiley.

JOHNSTON, M.J.S., 1997, Review of electrical and magnetic fields accompanying seismic and volcanic activity, Surv. Geophys., 18, 441-475.

JONES, A.G., OLAFSDOTTIR, B. AND TIIKKAINEN, J., 1983. Geomagnetic induction studies in Scandinavia III. Magnetotelluric observations, J. Geophys., 54, 35-50.

KHARIN, E.P., 1982. Changes in the transfer functions with time, Geophysical Surveys, 4, 467-500.

LARSEN, J.C., 1989. Transfer functions: Smooth robust estimates by least squares and remote reference methods, Geophys. J. Int., 99, 655-663.

MYACHKIN, V., BRACE, W., SOBOLEV, G. AND DIETERICH, J., 1975. Two models of earthquake forerunners, Pure Appl. Geophys., 113, 169-181.

KOUSKOUNA, V., 1991. Factors which modify the attenuation of seismic intensities in central Greece and contribution to the mapping of earthquake hazard, Doctorate Thesis, Univ. of Athens (in Greek, w. extended abstract in English).

PARK, S.K., JOHNSTON, M.J.S., MADDEN, T.R., MORGAN, F.D. AND MORISSON, H.F., 1993. Electromagnetic precursors to earthquakes in the ULF band: a review of observations and mechanisms, Rev. Geophys., 31, 117-132.

PEDERSEN, L.B., 1982. The magnetotelluric impedance tensor - its random bias and errors, Geophysical Prospecting, 30, 188-210.

ROZLUSKI, C.P., AND YUKUTAKE, T., (1993). Preliminary analysis of magnetotelluric and seismic activity in the Cxubu district, Japan, Acta Geophys. Polonica, 41, 17-26.

SCHOLZ, C.H., 1990. The Mechanics of Earthquakes and Faulting, Cambridge University Press, 439pp.

SIMS, W.S., BOSTICK, F.X., JR. AND SMITH, H.W., 1971. The estimation of magnetotelluric impedance tensor elements from measured datal, Geophysics, 36, 938-942.

SUTARNO, D. AND VOZOFF, K., 1991. Phase-smoothed robust M-estimation of magnetotelluric impedance functions, Geophysics, 56, 1999-2007.

SVETOV, B.S., KARINSKIJ, S.D., KUSKA, Y.I. AND ODINTSOV, V.I., 1997. Magnetotelluric monitoring of geodynamic processes, Annali di Geofisica, 40, 435-444.

TZANIS, A., 1988. Investigations on the properties and estimation of the Earth response operators from EM sounding data, PhD Thesis, Univ. of Edinburgh.

TZANIS, A. AND BEAMISH, D., 1989. A high resolution spectral study of audiomagnetotelluric data and noise interactions, Geophys. J., 97, 557-572.

TZANIS, A., 1994. MAGNET: A magnetotelluric data editing system, suitable for earthquake prediction research. Proceedings, XXIV ESC Gen. Assembly, Athens, Greece, 1994, Vol. I, 129-141. 